\title{
Sistema de registro de daños para determinar el estado constructivo en muros de adobe
}

\author{
María Cecilia Achig B. ${ }^{1}$, Marcelo Zúñiga L. ${ }^{1}(\dagger)$, Koenraad Van Balen ${ }^{2}$, Lourdes Abad R. ${ }^{3}$ \\ ${ }^{1}$ Investigador del proyecto World Heritage City Preservation Management, Facultad de Arquitectura y \\ Urbanismo de la Universidad de Cuenca, Cuenca, Ecuador. \\ ${ }^{2}$ Centrum voor Monumentenzorg, Katholieke Universiteit Leuven, Leuven, Belgium. \\ ${ }^{3}$ Consultor externo, Cuenca, Ecuador.
}

Autor para correspondencia: cecilia_achig@hotmail.com

Fecha de recepción: 10 de octubre 2013 - Fecha de aceptación: 28 de noviembre 2013

\section{RESUMEN}

Los muros de adobe son elementos comunes en la construcción de edificios patrimoniales en el Centro Histórico de Cuenca. La mayoría de estas edificaciones fueron construidas en la época republicana, especialmente durante los años 1860-1940. Las amenazas de origen natural y antrópico producen con el tiempo un deterioro de los edificios patrimoniales. Dentro de las actividades de conservación es importante tener una idea clara y precisa de la situación de daños y el estado constructivo de los muros de adobe. Con este fin, se propone generar un sistema de registro de daños que permita conocer el estado actual de muros de adobe en la ciudad de Cuenca. Estudios sobre la condición de muros de adobe, dieron como resultado la determinación de indicadores medibles y sus respectivas variables, que describen de forma cualitativa y cuantitativa el estado constructivo de los muros. Posteriormente, se desarrolló un sistema basado en una matriz de priorización y ponderación de las variables, a través del cual se aplicó un análisis multivariado que permite obtener un indicador integral que califica la condición del muro de adobe. Este indicador no solamente refleja la urgencia de intervención, sino también el tipo de intervención requerida o acciones de monitoreo para la conservación del muro.

Palabras claves: Edificación patrimonial, muro de adobe, daños, sistemas constructivos tradicionales, indicadores y variables, análisis multivariado.

\begin{abstract}
Adobe walls are common construction elements in the heritage buildings in the historic center of the City of Cuenca. Most of these buildings were constructed in the Republican period, especially in the period 1860-1940. Threats from natural and human origin resulted over time in a degradation of the heritage buildings. An important guide for the conservation is having a clear and accurate picture of the degraded status and condition of the adobe walls. To this end a damage registration system characterizing the current constructive state of the adobe walls in the City of Cuenca was developed. Surveys of the condition of adobe walls resulted in the derivation of measurable indicators and their respective variables, describing in a qualitative and quantitative way the constructive condition of the walls. A matrix based system for the prioritization and weighing of the variables was developed. Applying multivariate analysis resulted in the derivation of a global status indicator of the adobe wall. This indicator not only reflects the urgency of intervention, but also the type of constructional intervention or monitoring action required for the conservation of the wall.
\end{abstract}

Keywords: Heritage building, adobe wall, damage, traditional construction systems, status indicators and variables, multivariate analysis. 


\section{INTRODUCCIÓN}

La tierra ha sido el material de construcción predominante en casi todos los climas cálido-secos y templados del mundo. Las técnicas de construcción con barro datan de hace más de 9000 años. (Minke, 2001). Aún en la actualidad alrededor del 30\% de la población mundial vive en edificaciones de tierra, en países en vías de desarrollo esto representa alrededor del $50 \%$ y la mayor parte de la gente de las zonas rurales habita en viviendas de tierra (Fratini $y$ col., 2011).

En el actual territorio del Ecuador, la tierra como material de construcción fue utilizada desde épocas pre incásicas e incásicas. En la ciudad de Cuenca, con la llegada de los españoles se comenzaron a edificar modestas viviendas coloniales de las cuales se dice que eran como aquellas de España, construidas con piedra, lodo y adobe hechos de tierra... (Jaramillo, 2004). Con la emancipación de Cuenca y del Ecuador (1830), se produjeron cambios ideológicos, políticos, sociales, etc.; sin embargo, en cuanto a la arquitectura, se mantuvieron los materiales y técnicas tradicionales de construcción hasta 1860 aproximadamente. En esta época republicana, debido a las nuevas condiciones económicas -basadas principalmente en la exportación de sombreros de paja toquilla y la cascarilla- se generan vínculos directos con la economía y cultura europeas (Jaramillo, 2004). En este contexto, surgió la renovación de la arquitectura civil en Cuenca, tanto en su forma como en sus técnicas constructivas, a finales del siglo XIX y principios del siglo XX. Las viejas casas de adobe de una planta, son demolidas dando la posta a edificios de dos y tres plantas, con fachadas calcadas de las construcciones parisinas (Espinoza y Calle, 2002). Si bien en algunos casos se sustituyeron paulatinamente los gruesos muros de adobe por mampostería de ladrillo, (Espinoza y Calle, 2002) en la mayoría de las edificaciones se produjo una arquitectura de fachadas, es decir, se conservaron los muros de adobe, produciéndose cambios únicamente a nivel de fachada. Alrededor de 1940, la influencia del movimiento moderno en la arquitectura llegó a Cuenca a través del arquitecto uruguayo Gilberto Gatto Sobral, quien es el autor del Palacio Municipal, así como del Plan Regulador de Cuenca en 1948. En esta época, varias edificaciones del centro de Cuenca fueron sustituidas por las modernas (Achig y Paredes, 2001), acorde con las nuevas necesidades de la sociedad.

Hoy en día, en el Centro Histórico de la ciudad de Cuenca, declarado por la UNESCO en 1999 como Patrimonio Mundial de la Humanidad, la mayoría de sus edificaciones patrimoniales son construidas de tierra (adobe). En efecto, de acuerdo a los datos de inventario a nivel de registro (I. Municipalidad de Cuenca, 2010) del total de edificaciones inventariadas, el 43,2\% corresponde a fachadas de adobe y el 18,2\% tiene como estructura muros de adobe.

\subsection{Composición del adobe y principales propiedades}

El material con el que se fabrica el adobe es básicamente una mezcla de tierra seleccionada, agua y fibras. La tecnología varía de acuerdo a las costumbres y capacidades locales. Para fabricar adobes es recomendable el uso de tierra areno-arcillosa con poco limo. Si la tierra tiene mucha arcilla, aumenta el riesgo de fisuración al secarse; si tiene demasiada arena o limo, puede carecer de cohesión interna adecuada y desgranar fácilmente, además de que disminuye su resistencia a compresión (Rotondaro, 2011). Hay gran diversidad de opiniones en cuanto a los porcentajes adecuados de cada componente. La norma peruana NTE E 080 (SENCICO, 2000 en Rotondaro, 2011) propone los siguientes porcentajes en volumen para la tierra: arcilla $10 \%$ a $20 \%$, limo $15 \%$ a $25 \%$, y arena $55 \%$ a $70 \%$. Adicionalmente, se utilizan otros materiales como fibras vegetales (paja), estiércol y/o pelos de animal para evitar la fisuración por secado. El uso de la paja además reduce la resistencia a la compresión (Binici $y$ col., 2005 en Pacheco y Said Jalali, 2011).

En general, la resistencia a compresión del adobe se encuentra entre 1,3 $\mathrm{Mpa}^{1}$ y 2,1 MPa. (Jiménez y Cañas, 2007). Sin embargo, para lograr una óptima estabilización del suelo, es decir cambiar sus características con el fin de mejorar su comportamiento mecánico o físico, se sugiere que la resistencia a la comprensión oscile entre 2,0 MPa y 2,5 MPa (Houben y Guillaud, 1989). En Cuenca se han realizado varias pruebas para medir la resistencia a la compresión de adobes y se ha obtenido

\footnotetext{
${ }^{1} 1 \mathrm{Mpa}=10 \mathrm{~kg} \mathrm{~cm}^{-2}$
} 
resultados desde 1,3 MPa en adobes utilizados para la restauración de la Escuela La Inmaculada (Campos, 2009) hasta 1,5 MPa y 1,79 MPa en cilindros de adobe provenientes de Sinincay (De Jongh y Van Wijnendaele, 2009). Como se observa hay una buena relación entre la literatura y los resultados obtenidos en varios adobes que se están utilizando en Cuenca. La resistencia a la tracción del adobe es baja, el adobe trabaja mejor a compresión. Para mejorar la resistencia a la tracción, se añaden fibras vegetales (paja) a la mezcla de tierra. La resistencia a la tracción es difícil de medir debido a los efectos de las grietas de contracción; sin embargo, oscila entre 0,370 a 0,834 MPa (Brown y Clifton, 1978).

El tamaño de los adobes es variable, las dimensiones dependen mucho de la manera tradicional de construir y de la experiencia del constructor. En el Ecuador se pueden encontrar adobes con dimensiones que van entre los siguientes rangos: largo $25-60 \mathrm{~cm}$, ancho $14-20 \mathrm{~cm}$ y altura $10-17 \mathrm{~cm}$ (De Jongh y Van Wijnendaele, 2009). Por otra parte, la tierra tiene la capacidad de absorber y desorber humedad más rápido y en mayor cantidad que los demás materiales de construcción (Minke, 2001). Por eso regula el clima interior y ofrece mayor confort a las personas que habitan en edificaciones de tierra. Existen otras propiedades fundamentales del adobe, que deben ser abordadas a profundidad dependiendo del tipo de estudio que se realice. Estas propiedades son: textura, plasticidad, compactibilidad y cohesión (Houben y Guillaud, 1989).

\subsection{Causas de deterioro en muros de adobe}

Las construcciones históricas de adobe casi siempre presentan grietas generadas por actividad sísmica anterior, por deformación o por asentamiento de la cimentación (Leroy Tolles y col., 2002). Las edificaciones de adobe en el Centro Histórico de Cuenca a través del tiempo han tenido que hacer frente a cargas sísmicas. En 1887 se produjo en la provincia del Azuay un gran sismo (Rivera y Rivera, 2001) y desde esa fecha ha habido una serie de pequeños sismos que han afectado a las edificaciones. Esas cargas sísmicas son absorbidas dependiendo de la condición de las estructuras de adobe. Los sismos aceleran los daños que ya se han manifestado en las construcciones de adobe. Por otra parte, el adobe sufre una dramática reducción de resistencia cuando se moja. Las fuerzas de compresión y tracción pueden reducirse entre un 50\% y un 90\% (Leroy Tolles y col., 2002). El adobe con la presencia de humedad pierde sus propiedades de cohesión.

Las fallas constructivas, deficiente mantenimiento y malas intervenciones, afectan a la estabilidad y aceleran la evolución de daños en edificios con muros de adobe, pudiéndole llevar incluso a una situación de colapso. Los principales daños que podrían llevar al muro de adobe a un estado de colapso son los siguientes: grieta de flexión, grieta y falla de tímpano, grietas horizontales a mediana altura, grietas de corte en forma de X, grietas verticales en las esquinas, grietas diagonales en las esquinas, grietas en forma de aspas en las esquinas, grietas en aberturas, grietas entre muros perpendiculares (Leroy Tolles y col., 2002), grietas en forma de curva parabólica (Piedra, 2008), desplome de muros, erosión basal y erosión del cabezal del muro.

\subsection{La importancia de conocer el estado constructivo de un muro de adobe para la conservación}

Para la conservación del patrimonio, resulta necesario conocer el estado constructivo que presentan los muros de adobe, así como el valor de la arquitectura patrimonial. La autenticidad es según Van Balen (2007) un aspecto importante que debe ser considerado dentro del tema de valores en arquitectura. De acuerdo con el Documento de Nara, la autenticidad y valores se definen por la fuente de información de un bien patrimonial en los siguientes aspectos: forma y diseño, materiales y substancia, uso y función, tradiciones, técnicas y experticia, lugares y asentamientos, espíritu y sentimiento (The Nara Documenton Authenticity, 1994). En el documento de Nara se insiste en la veracidad y posibilidad de verificar la información que da sustento a condiciones de integridad y autenticidad. En este contexto, el análisis del daño está relacionado con la condición física de un edificio. El valor tecnológico determina que es necesario emplear materiales y técnicas tradicionales de construcción no sólo para optimizar recursos, sino porque en ellas se refleja una relación particular con la historia (García, 2000). La conservación de edificios patrimoniales implica la conservación de la materia, pues el valor tecnológico y la autenticidad están determinados por ésta. Por ello, se debe conservar los muros de adobe que dan mayor valor a las edificaciones patrimoniales. Existe una relación entre los valores 
patrimoniales y la forma de intervenir cuando se presenta un daño. Se puede seguir el siguiente enfoque no lineal y consecutivo de intervención: análisis, diagnóstico, tratamiento y control (Van Balen, 2007).

El conocimiento de los daños en muros de adobe es importante también por las demandas de información por parte de las instituciones y por parte de los particulares para determinar las intervenciones. La sistematización mediante ordenadores es actualmente la única manera de poder manejar la información de forma ágil y eficientemente.

Por lo escrito previamente, se espera que el proceso sistemático para determinar el estado constructivo de muros de adobe mejore considerablemente el tiempo y el tipo de intervención para logar una oportuna recuperación de los edificios patrimoniales que tienen muros de adobe como elementos estructurales. En este estudio se identificaron una serie de indicadores y variables cualitativas y cuantitativas que afectan el estado constructivo de un muro de adobe y aceleran su deterioro. Para simplificar la interpretación integrada de la condición global del estado constructivo del muro de adobe, se desarrolló una matriz de priorización y ponderación de las variables, de la cual se derivó un indicador integral utilizando un análisis multivariado. Finalmente, en el manuscrito se realiza una descripción completa de las etapas sucesivas para llegar a la determinación del indicador integral del estado constructivo del muro de adobe.

\section{MATERIALES}

Las siguientes fuentes de información sobre daños en muros de adobe fueron consultadas para la investigación:

a) Los inventarios de 1975, 1980 y 1999 de la ciudad de Cuenca y los datos generales de los proyectos de restauración (Proyecto vlirCPM World Heritage City Perservation Management, 2009).

b) Varias tesis de maestría entre las cuales, para fines de la investigación, se destacan: Earthquakes and other damaging mechanisms to earth structures, Cuenca, Ecuador (De Jongh y Van Wijnendaele, 2009; De Jongh y col., 2009); Patologías y sus causas en muros en tierra cruda y cocida en las edificaciones patrimoniales (Piedra, 2008); y Methodology for analysis, diagnosis and monitoring of damage in heritage architecture (earth and timber) in Cuenca, Ecuador: Case Study Casa Peña in the Barranco of the city (Achig, 2010). En estas tres investigaciones se analiza de manera general el tema de daños en muros de adobe y además no se consideran los factores y variables que inciden sobre el estado constructivo del muro de adobe y que aceleran el deterioro, lo cual es fundamental para entender el comportamiento de las estructuras de tierra.

c) El Atlas de Daños para las edificaciones de Cuenca (Proyecto vlirCPM, 2010), ofrece una guía para la identificación de daños y sus posibles causas en edificaciones patrimoniales. Los daños se clasifican de acuerdo a su tipología e intensidad en: alteraciones cromáticas, degradación y desprendimiento, fisuras y/o grietas y deformación. En este documento se registran daños presentes en todos los elementos constitutivos de un bien patrimonial, incluyendo los muros de adobe.

A partir de esta información, el presente artículo desarrolla un sistema de registro de daños para determinar los factores y variables que inciden en el estado constructivo del muro y que aceleran el deterioro de los daños. Si bien estos documentos sirven como base, no existe una sistematización de la información para determinar el estado constructivo de un muro de adobe, lo cual constituye el alcance de la presente investigación. A pesar de que a nivel internacional existen numerosas instituciones que han trabajado sobre la arquitectura de tierra, como la Red Iberoamericana PROTERRA, CRAterre, el Instituto Getty de Conservación, ICCROM, entre otras, no existe un método estandarizado para el registro del estado constructivo de muros de adobe. 
Además de la limitada cantidad y calidad de información sobre el registro de daños, dispersa y disponible en distintas instituciones; problemas que obstaculizan el procesamiento y la interpretación de la información disponible, se pueden resumir según Carreño (2006) en:

a) El carácter incompleto de la información. En los inventarios no se establecen los mecanismos y las causas que generan los daños en edificios históricos, y no hay ninguna información sobre los cambios o afectaciones en las edificaciones patrimoniales a lo largo del tiempo (Proyecto vlirCPM, 2009).

b) Falta de conocimiento, entrenamiento y experiencia de los evaluadores. Para llevar a cabo un buen proceso de evaluación de daños, es importante contar con evaluadores con amplia experiencia y experticia. Sin embargo, cuando se trata de procesos más amplios como el caso de inventarios de las edificaciones patrimoniales existentes en conjuntos o centros históricos; los daños en la zona pueden ser tan generalizados que no es posible que los expertos se encarguen de hacer la totalidad de las evaluaciones.

c) Subjetividad en las evaluaciones. Los niveles de daño son definidos en la mayoría de los métodos de evaluación con calificaciones como leve, menor, moderado, medio, severo, grave o fuerte; conceptos que pueden tener una notable variación en su significado según la persona y experiencia de quien los utilice. Lo que para una persona es moderado, para otra puede ser severo; así como puede estar en medio de los dos conceptos para otra. Por esto, es necesario intentar definir los criterios y volver a la evaluación lo más cuantitativa posible, determinando porcentaje de elementos afectados, tamaño, tipo de daño, etc.

d) Falta de organización y sistematización del inventario de daños. Mantener un registro del procedimiento de evaluación de daños constituye una tarea de gran importancia para la conservación y actuación en la edificación patrimonial. Normalmente, no se le ha dado el valor que merece a esta parte del proceso, lo cual ha provocado desinformación, confusiones y retrasos en la toma de decisiones. Falta también la opción de actualizar los inventarios, y las recomendaciones para prevenir los daños en los proyectos de restauración en edificaciones patrimoniales son muy limitadas. (Achig, 2010).

e) Falta de un sistema que registre los daños en muros de adobe de una manera sistemática, organizada con resultados que puedan ser monitoreados a futuro. Esto muestra además la falta de valoración patrimonial en relación con los materiales de las edificaciones del Centro Histórico de Cuenca.

\section{METODOLOGÍA}

La evaluación del estado en el que se encuentra una edificación patrimonial debe considerar los diferentes elementos que la constituyen, tales como: elementos estructurales, elementos no estructurales, condiciones del suelo y condiciones preexistentes. Los tres primeros indican el estado del edificio que puede comprometer la existencia del bien inmueble patrimonial. Las condiciones preexistentes se relacionan con la calidad de los materiales de construcción, la configuración estructural, la geometría/proporción del edificio y con el mantenimiento; estas características son útiles para estudiar la reparabilidad del edificio (Carreño, 2006).

A nivel internacional, existen dos investigaciones donde se refleja la idea de presentar resultados objetivos para la evaluación de daños. Se trata de la tesis doctoral Técnicas innovadoras para la evaluación del riesgo sísmico y su gestión en centros urbanos: Acciones ex ante y ex post de la autora Carreño (2006), donde se propuso un modelo de evaluación de daños en edificios después de un terremoto, basado en técnicas de inteligencia computacional como redes neuronales artificiales y conjuntos difusos; este modelo hace posible una evaluación más exacta de un daño estructural y el riesgo asociado a éste, a través de resultados cualitativos y cuantitativos. Otro estudio que propone una metodología para la evaluación del nivel y magnitud del riesgo, es patrocinado por la UNESCO: Risk management at heritage sites. A case study of the Petra world heritage site (Paolini y col., 2012), el cual se basa en enfoques cualitativos y cuantitativos planteando la sumatoria de tres criterios: (i) 
probabilidad o magnitud del daño; (ii) pérdida de valores e integridad; y (iii) porcentaje del área evaluada susceptible a la amenaza y su vulnerabilidad.

El modelo propuesto en la presente investigación establece los principales factores que tienen incidencia sobre el estado constructivo del muro, expresados en un conjunto de variables que representan las condiciones del muro y que a su vez son susceptibles de ser identificadas, medidas o cuantificadas. El sistema planteado emplea el análisis multivariado (Malczewski, 1999) para interrelacionar apropiadamente distintas variables y obtener un indicador integrado. Se trata de un método de priorización de variables basado en matrices. La metodología se sintetiza principalmente en las siguientes partes: (i) definición de indicadores y variables que caracterizan el estado constructivo del muro de adobe; (ii) asignación de valores cualitativos y cuantitativos a las variables; y (iii) priorización y ponderación de variables.

A través de la combinación de las variables (Tabla 1), esta investigación desarrolla el sistema para evaluar el muro de adobe. En efecto, el modelo propuesto establece los principales factores que tienen incidencia sobre el estado constructivo del elemento y son: sistema constructivo, geometría del elemento, acciones mecánicas y medio ambiente. Estos indicadores se relacionan directamente con las variables, puesto que para la formulación de las mismas, se realizó un estudio exhaustivo del sistema constructivo de un muro de adobe, su geometría, acciones mecánicas que lo puedan afectar y cómo el ambiente incide sobre el mismo. El conjunto de variables representa las condiciones del muro y son susceptibles de ser identificadas, medidas o cuantificadas, descritas, documentadas y valoradas, en términos de su estado de afectación.

\subsection{Asignación de valores cualitativos y cuantitativos a las variables}

Se asigna una calificación dependiendo del daño observado utilizando entre tres y cinco posibles niveles de daño en función de la variabilidad, en primer término se establece una escala cualitativa. La Tabla 2 ilustra las denominaciones utilizadas por el modelo para estas calificaciones. Luego se asocia para cada condición que presenta la variable un valor cuantitativo, lo cual permitirá aplicar un proceso matemático para determinar de forma integrada un indicador de estado que sintetiza el análisis del muro y por consiguiente permite obtener una valoración global del elemento. La escala cuantitativa indica el nivel de daño en el muro de adobe que va desde 0 hasta 1 . El valor 0 indica que se cumple con la condición presentada en la variable o que el elemento no presenta daños; el valor 0,25 indica la presencia de un daño en bajo nivel; los valores 0,5 y 0,75 indican que se está próximo al límite y finalmente el valor 1 muestra que el límite de la variable ha sido superado y por lo tanto existe un daño eminente. Lo indicado se muestra en la Tabla 2.

\subsection{Priorización y ponderación de las variables}

En la edificación, la situación real de un muro podría presentar de forma simultánea varias condiciones de afectación para las 20 variables definidas, de las cuales: las referidas a magnitud y si el muro es de carga o no, se consideran como factores de incidencia general; y, por consiguiente, influyen de forma directa en la calificación del estado general del elemento muro. Es decir, se empieza con 20 variables, pero dos de ellas van a formar parte del nivel de gravedad del muro, que se explicará más adelante. Por esta razón, la matriz se estructura con 18 variables.

Posteriormente, se procede a determinar los grados $(0 ; 0,25 ; 0,5 ; 0,75 ; 1)$ de cada una de las relaciones entre las variables. Los grados establecen en qué medida influye o depende un atributo hacia los otros. El grado 1 indica que una variable es determinante o agravante con respecto a otras, por ejemplo: (1) La esbeltez y grosor del muro es determinante o incide totalmente en (14) el grado de inclinación del muro. El grado 0,75 señala que una variable tiene alta incidencia en relación a otras; esta incidencia va disminuyendo en el grado 0,5 y 0,25 hasta llegar al grado 0 , en donde no existe relación alguna entre las variables. Esta fase del proceso constituye la parte más importante en el modelo empleado, puesto que determina en qué medida las variables aportan de manera individual e integrada a la calificación global del muro de adobe. Por esta razón, se procedió a consultar a un conjunto de expertos para construir la matriz de priorización de variables. Como resultado de esta actividad se obtuvieron los resultados que se presentan en la Tabla 3. 


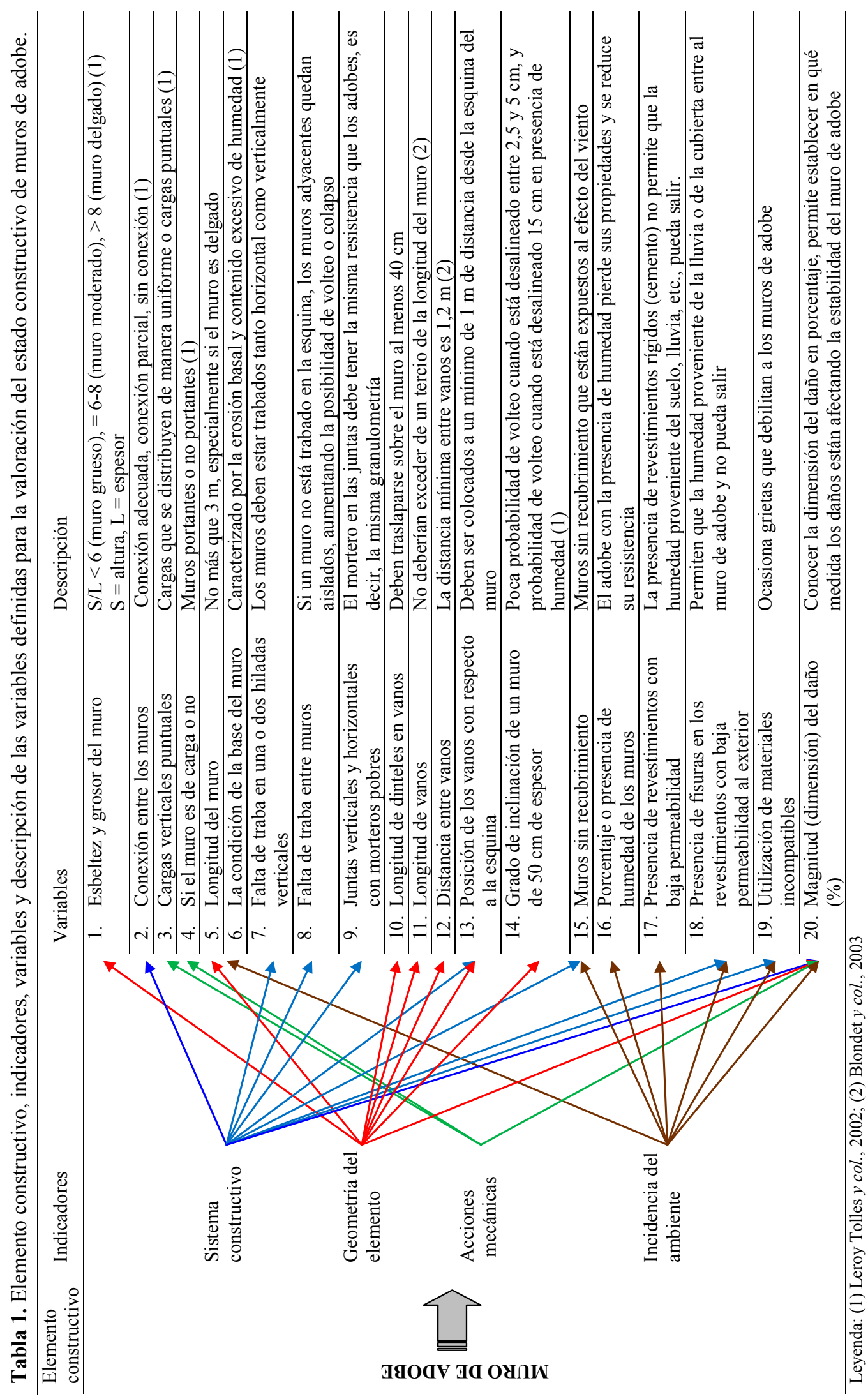




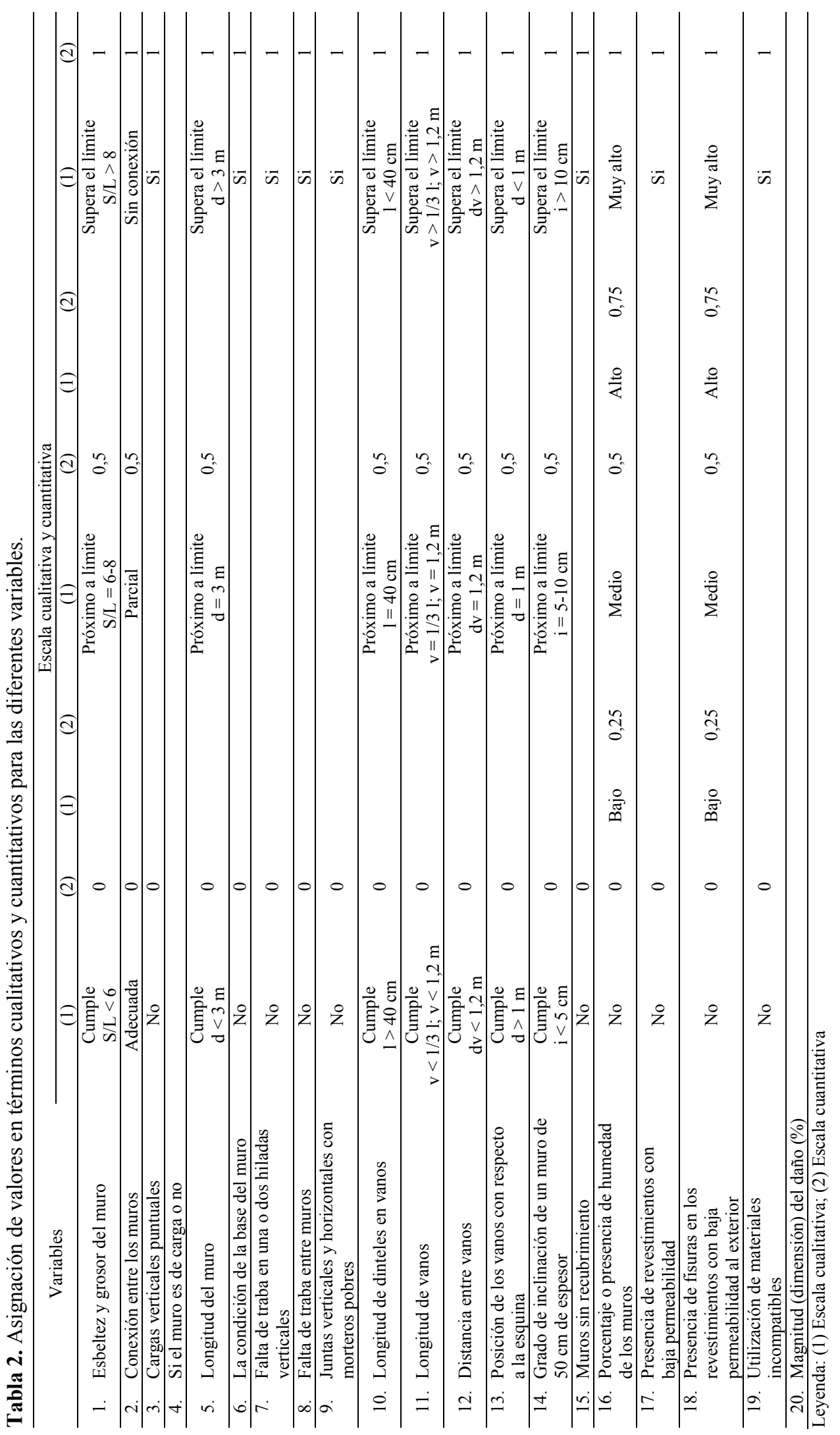




\begin{tabular}{|c|c|c|c|c|c|c|c|c|c|c|c|c|c|c|c|c|c|c|c|c|}
\hline 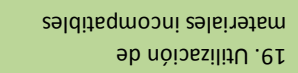 & $\left|\begin{array}{l}0 \\
h \\
0 \\
0\end{array}\right|$ & $\begin{array}{l}\text { 员 } \\
\text { o }\end{array}$ & $\left|\begin{array}{l}0 \\
0 \\
0 \\
0\end{array}\right|$ & $\left|\begin{array}{l}0 \\
0 \\
0 \\
0\end{array}\right|$ & $\begin{array}{l}\stackrel{2}{2} \\
0 \\
0\end{array}$ & $\stackrel{\stackrel{n}{\sim}}{\sigma}$ & $\stackrel{\stackrel{\sim}{n}}{\circ}$ & $\stackrel{\stackrel{\leftrightarrow}{\sim}}{\sigma}$ & $\stackrel{\stackrel{\mu}{N}}{0}$ & $\stackrel{\substack{n \\
0}}{0}$ & $\left|\begin{array}{c}\stackrel{N}{N} \\
0 \\
0\end{array}\right|$ & 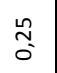 & $\left|\begin{array}{l}\hat{n} \\
\hat{0}\end{array}\right|$ & 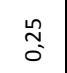 & $\left|\begin{array}{l}\hat{n} \\
\hat{0} \\
0\end{array}\right|$ & $\begin{array}{l}\text { ñ } \\
\text { o }\end{array}$ & $\mid \begin{array}{c}n \\
\hat{\sigma}\end{array}$ & $\times$ & $\stackrel{n}{\sim}$ & $\begin{array}{l}m \\
0 \\
0 \\
0 \\
0 \\
0\end{array}$ \\
\hline 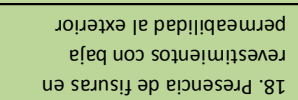 & $\left.\mid \begin{array}{c}n \\
0 \\
0\end{array}\right]$ & 员 & 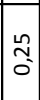 & $\begin{array}{c}\stackrel{n}{N} \\
0 \\
0\end{array} \mid$ & $\begin{array}{l}n \\
\hat{0} \\
0\end{array}$ & $\stackrel{\stackrel{n}{0}}{0}$ & 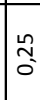 & $\stackrel{\stackrel{n}{\simeq}}{0}$ & $\stackrel{\substack{n \\
0 \\
0}}{n}$ & $\begin{array}{l}\stackrel{\sim}{n} \\
0 \\
0\end{array}$ & $\begin{array}{l}\stackrel{n}{N} \\
0 \\
0\end{array} \mid$ & $\begin{array}{l}\stackrel{\text { N}}{\tilde{O}} \\
\text { - }\end{array}$ & $\left|\begin{array}{l}0 \\
0 \\
0 \\
0\end{array}\right|$ & 贶 & $\left|\begin{array}{l}8 \\
- \\
-i\end{array}\right|$ & $\begin{array}{l}\text { ñ } \\
\text { o }\end{array}$ & $x$ & $\begin{array}{l}n \\
2 \\
0 \\
0\end{array} \mid$ & $\stackrel{\stackrel{n}{N}}{=}$ & $\begin{array}{l}m \\
0 \\
\\
0 \\
0\end{array}$ \\
\hline 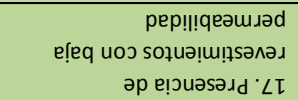 & {$\left[\begin{array}{l}\stackrel{n}{\sim} \\
0 \\
0\end{array}\right]$} & 㔛 & 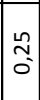 & $\left|\begin{array}{c}\stackrel{2}{2} \\
0 \\
0\end{array}\right|$ & $\begin{array}{l}n \\
\vdots \\
0 \\
0\end{array}$ & $\stackrel{\stackrel{\sim}{N}}{\stackrel{0}{0}}$ & $\stackrel{\text { L }}{\circ}$ & $\stackrel{\stackrel{\leftrightarrow}{0}}{\sigma_{0}^{\prime}}$ & $\stackrel{\stackrel{n}{n}}{0}$ & $\left|\begin{array}{c}\stackrel{n}{2} \\
0 \\
0\end{array}\right|$ & $\left|\begin{array}{c}\stackrel{N}{N} \\
0 \\
0\end{array}\right|$ & 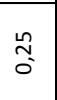 & $\left|\begin{array}{l}0 \\
0 \\
0 \\
0\end{array}\right|$ & $\begin{array}{l}\text { 茴 } \\
\text { - }\end{array}$ & $\left|\begin{array}{l}n \\
\hat{0} \\
0\end{array}\right|$ & $x$ & $\begin{array}{l}\hat{n} \\
\hat{0}\end{array}$ & $\begin{array}{c}n \\
\hat{0} \\
0\end{array} \mid$ & $\stackrel{8}{:}$ & $\mid \begin{array}{ll}n \\
\\
0 \\
0 \\
0\end{array}$ \\
\hline 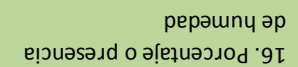 & $\left|\begin{array}{c}n \\
0 \\
0\end{array}\right|$ & 员 & $\mid \begin{array}{l}0 \\
\text { no } \\
0\end{array}$ & $\left|\begin{array}{l}0 \\
h \\
0 \\
0\end{array}\right|$ & $\begin{array}{l}n \\
\vdots \\
0\end{array}$ & $\stackrel{\stackrel{\sim}{\sim}}{\stackrel{0}{0}}$ & $\begin{array}{l}\stackrel{2}{2} \\
0 \\
0\end{array}$ & 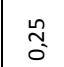 & 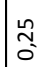 & $\mid \begin{array}{l}0 \\
\substack{n \\
0 \\
0}\end{array}$ & $\left|\begin{array}{c}n \\
N \\
0\end{array}\right|$ & $\begin{array}{l}\stackrel{\sim}{n} \\
\stackrel{0}{0}\end{array}$ & $\left|\begin{array}{c}n \\
\hat{0} \\
0\end{array}\right|$ & $\begin{array}{l}\text { 总 } \\
\text { c. }\end{array}$ & $\times$ & $\begin{array}{l}\frac{n}{2} \\
0 \\
0\end{array}$ & $\underset{i}{8}$ & $\left|\begin{array}{c}n \\
\vdots \\
0\end{array}\right|$ & $\begin{array}{c}n \\
\infty \\
\infty\end{array}$ & 䍃 \\
\hline 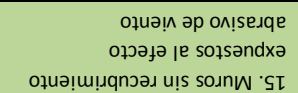 & $\left.\begin{array}{c}\stackrel{n}{N} \\
0 \\
0\end{array}\right]$ & 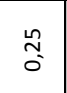 & $\left|\begin{array}{l}0 \\
0 \\
0\end{array}\right|$ & 竞 & $\begin{array}{l}n \\
\vdots \\
0\end{array}$ & $\stackrel{\stackrel{n}{\sim}}{0}$ & $\stackrel{\stackrel{N}{N}}{0}$ & 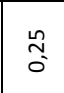 & $\begin{array}{l}\stackrel{\sim}{\sim} \\
0 \\
0\end{array}$ & $\begin{array}{l}\stackrel{\sim}{N} \\
0 \\
0\end{array}$ & {$\left[\begin{array}{l}n \\
N \\
0 \\
0\end{array}\right]$} & 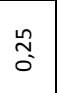 & $\mid$ & $x$ & $\begin{array}{l}\stackrel{0}{n} \\
0 \\
0\end{array} \mid$ & 员 & 总 & $\begin{array}{c}\stackrel{\sim}{N} \\
0 \\
0\end{array} \mid$ & $\begin{array}{l}\stackrel{\sim}{n} \\
\text {. }\end{array}$ & $\mid \begin{array}{l}0 \\
\infty \\
0 \\
0 \\
0\end{array}$ \\
\hline 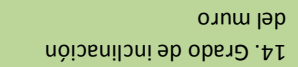 & 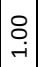 & $\begin{array}{l}n \\
\hat{o} \\
o\end{array}$ & $\mid$ & $\mid$ & $\underset{-}{8}$ & $\stackrel{\stackrel{\sim}{N}}{0}$ & $\begin{array}{l}\hat{n} \\
\hat{0}\end{array}$ & 号 & $\left|\begin{array}{l}n \\
\hat{0} \\
\delta\end{array}\right|$ & \begin{tabular}{|l|l}
$n$ \\
$\vdots$ \\
0
\end{tabular} \mid & $\mid$\begin{tabular}{l|l}
$n$ \\
$\hat{0}$ \\
0
\end{tabular} & $\stackrel{n}{\stackrel{n}{o}}$ & $\times$ & 号 & $\left|\begin{array}{l}n \\
\hat{o} \\
0\end{array}\right|$ & 员 & 号 & $\left|\begin{array}{l}n \\
\hat{0} \\
0\end{array}\right|$ & $\stackrel{\sim}{\sim}$ & 竞 \\
\hline 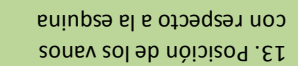 & $\left|\begin{array}{c}n \\
\hat{0} \\
0\end{array}\right|$ & $\begin{array}{l}\stackrel{0}{n} \\
\text { o. }\end{array}$ & $\left|\begin{array}{l}0 \\
0 \\
0 \\
0\end{array}\right|$ & 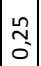 & $\begin{array}{c}n \\
\tilde{c} \\
0\end{array}$ & $\stackrel{\stackrel{\sim}{\sim}}{\stackrel{0}{0}}$ & $\begin{array}{l}\stackrel{\text { s. }}{\circ} \\
0\end{array}$ & $\stackrel{\substack{\text { In } \\
0}}{0}$ & $\left|\begin{array}{c}n \\
0 \\
0\end{array}\right|$ & \begin{tabular}{c}
$n$ \\
\multirow{2}{0}{} \\
0
\end{tabular} \mid & $\left|\begin{array}{c}n \\
\hat{0} \\
0\end{array}\right|$ & $x$ & $\left|\begin{array}{c}n \\
\hat{0} \\
0\end{array}\right|$ & $\begin{array}{l}\stackrel{n}{\underline{n}} \\
0\end{array}$ & 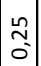 & $\stackrel{\substack{\tilde{O} \\
0}}{0}$ & $\underset{\sim}{\sim}$ & $\begin{array}{c}n \\
0 \\
0\end{array} \mid$ & $\stackrel{\sim}{N}$ & $\begin{array}{l}m \\
0 \\
0 \\
0\end{array}$ \\
\hline 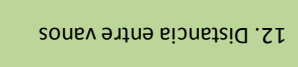 & 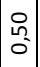 & 号 & $\mid \begin{array}{l}0 \\
0 \\
0 \\
0\end{array}$ & 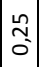 & $\begin{array}{c}\stackrel{n}{\sim} \\
\sigma^{\prime}\end{array}$ & $\stackrel{\stackrel{n}{n}}{o}$ & $\stackrel{\stackrel{n}{n}}{\sigma_{0}}$ & $\stackrel{\stackrel{\sim}{N}}{0}$ & $\left|\begin{array}{l}n \\
\hat{\sigma} \\
0\end{array}\right|$ & $\begin{array}{l}n \\
\hat{a} \\
0\end{array}$ & $\times$ & $\stackrel{n}{n}$ & $\mid \begin{array}{l}n \\
\hat{\alpha} \\
0\end{array}$ & $\underset{\sim}{\tilde{N}}$ & $\begin{array}{c}n \\
\tilde{N} \\
0\end{array}$ & 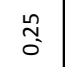 & $\stackrel{\sim}{\sim}$ & $\left|\begin{array}{c}\stackrel{n}{n} \\
0 \\
0\end{array}\right|$ & : & $\mid$ \\
\hline soue^ әр pn!!8uo7 'I I & 虽 & 员 & $\mid \begin{array}{l}0 \\
\text { hn } \\
0 \\
0\end{array}$ & $\left|\begin{array}{c}n \\
\tilde{O} \\
0\end{array}\right|$ & $\begin{array}{l}\stackrel{n}{\tilde{O}} \\
\sigma^{\prime}\end{array}$ & $\stackrel{\stackrel{n}{\sim}}{\circ}$ & $\stackrel{\stackrel{n}{\sim}}{\circ}$ & $\stackrel{n}{\stackrel{n}{0}}$ & $\mid \begin{array}{c}8 \\
-i\end{array}$ & $\times$ & $\left|\begin{array}{ll}n \\
\hat{0} \\
0\end{array}\right|$ & $\stackrel{n}{n}$ & $\left|\begin{array}{l}n \\
\hat{\sigma} \\
0\end{array}\right|$ & $\stackrel{\sim}{\sim}$ & $\mid \begin{array}{l}0 \\
\text { hon } \\
0\end{array}$ & $\stackrel{n}{\tilde{O}}$ & $\stackrel{\sim}{\sim}$ & $\begin{array}{l}n \\
\tilde{O} \\
0\end{array}$ & 员 & 贷 \\
\hline 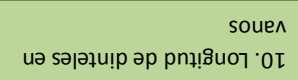 & 贻 & $\begin{array}{l}\text { 员 } \\
\text { on }\end{array}$ & $\mid \begin{array}{l}0 \\
0 \\
0 \\
0\end{array}$ & $\stackrel{\substack{n \\
0 \\
0}}{\mid}$ & $\begin{array}{l}\stackrel{n}{n} \\
0 \\
0\end{array}$ & $\stackrel{\stackrel{\sim}{N}}{\stackrel{0}{0}}$ & 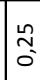 & $\stackrel{\stackrel{\sim}{0}}{0}$ & $\times$ & $\underset{i}{\stackrel{-}{-}}$ & $\left|\begin{array}{l}n \\
0 \\
0\end{array}\right|$ & 告 & $\left|\begin{array}{l}n \\
\hat{0} \\
0\end{array}\right|$ & $\begin{array}{l}\stackrel{n}{\tilde{O}} \\
\stackrel{0}{0}\end{array}$ & $\left|\begin{array}{c}\stackrel{n}{0} \\
0 \\
0\end{array}\right|$ & $\begin{array}{l}\stackrel{n}{\tilde{O}} \\
\stackrel{0}{2}\end{array}$ & 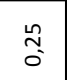 & $\stackrel{\substack{n \\
0 \\
0}}{0}$ & $\stackrel{\sim}{\sim}$ & \begin{tabular}{|l|}
0 \\
$\tilde{n}$ \\
0 \\
0 \\
0
\end{tabular} \\
\hline 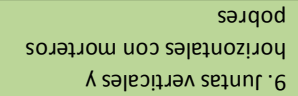 & 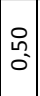 & $\begin{array}{l}\text { 员 } \\
\text { on }\end{array}$ & $\left|\begin{array}{l}0 \\
\text { hू } \\
0\end{array}\right|$ & $\mid \begin{array}{l}0 \\
\text { ho } \\
0\end{array}$ & $\begin{array}{l}\tilde{n} \\
\tilde{O} \\
0\end{array}$ & $\stackrel{\stackrel{n}{\sim}}{0}$ & 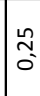 & $\times$ & $\left|\begin{array}{l}\stackrel{2}{2} \\
0 \\
\mid\end{array}\right|$ & $\begin{array}{l}n \\
N \\
0 \\
0\end{array}$ & {$\left[\begin{array}{l}\stackrel{n}{N} \\
0 \\
0\end{array}\right]$} & 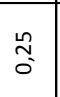 & $\mid \begin{array}{l}\text { 号 } \\
0 \\
0\end{array}$ & 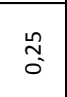 & $\left|\begin{array}{c}\tilde{N} \\
0 \\
0\end{array}\right|$ & $\begin{array}{l}\stackrel{n}{N} \\
0 \\
0\end{array}$ & 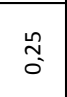 & $\left|\begin{array}{c}\stackrel{n}{2} \\
0\end{array}\right|$ & $\begin{array}{l}\stackrel{\sim}{\sim} \\
\text {. }\end{array}$ & $\left|\begin{array}{ll}0 \\
0 \\
0 \\
0 \\
0\end{array}\right|$ \\
\hline 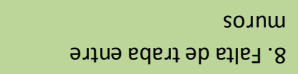 & $\left|\begin{array}{l}n \\
\hat{o} \\
0\end{array}\right|$ & 员 & 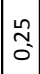 & $\begin{array}{c}n \\
\hat{0} \\
0\end{array} \mid$ & $\begin{array}{l}\text { 足 } \\
\text { on. }\end{array}$ & $\stackrel{\stackrel{\sim}{N}}{\tilde{O}^{\circ}}$ & $x$ & $\stackrel{\sim}{\sim}$ & $\left|\begin{array}{l}n \\
\hdashline \\
0\end{array}\right|$ & $\stackrel{\substack{n \\
0}}{0}$ & 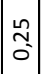 & $\stackrel{n}{\sim}$ & $\left|\begin{array}{c}n \\
\hat{\sigma} \\
0\end{array}\right|$ & $\begin{array}{l}\stackrel{\tilde{n}}{\tilde{O}} \\
\stackrel{0}{0}\end{array}$ & $\left|\begin{array}{c}n \\
\sim \\
0\end{array}\right|$ & $\begin{array}{l}\stackrel{n}{\tilde{O}} \\
\stackrel{0}{0}\end{array}$ & $\stackrel{\sim}{\sim}$ & $\left|\begin{array}{c}n \\
2 \\
0\end{array}\right|$ & 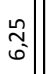 & $\left|\begin{array}{l}0 \\
0 \\
0 \\
0 \\
0\end{array}\right|$ \\
\hline 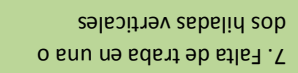 & $\left|\begin{array}{l}0 \\
h \\
0\end{array}\right|$ & 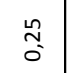 & $\left|\begin{array}{l}\stackrel{n}{N} \\
0\end{array}\right|$ & $\left|\begin{array}{c}\tilde{N} \\
0 \\
0\end{array}\right|$ & 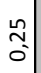 & $\times$ & $\stackrel{\text { In }}{\circ}$ & 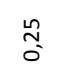 & $\left|\begin{array}{l}\stackrel{\sim}{2} \\
0 \\
0\end{array}\right|$ & $\left|\begin{array}{l}\stackrel{\sim}{2} \\
0 \\
0\end{array}\right|$ & 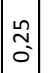 & $\stackrel{\stackrel{n}{N}}{\sigma}$ & $\left|\begin{array}{c}\stackrel{n}{2} \\
0 \\
0\end{array}\right|$ & $\begin{array}{l}\stackrel{n}{N} \\
\text { O }\end{array}$ & $\mid \begin{array}{l}\stackrel{n}{\hat{\alpha}} \\
0\end{array}$ & 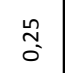 & 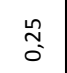 & $\left|\begin{array}{l}\stackrel{\sim}{2} \\
0 \\
0\end{array}\right|$ & $\begin{array}{l}\text { h̊ } \\
\text { q }\end{array}$ & $\left|\begin{array}{l}\vec{p} \\
0 \\
0 \\
0\end{array}\right|$ \\
\hline 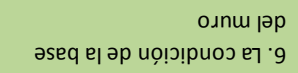 & $\left|\begin{array}{l}\hat{n} \\
\hat{o} \\
0\end{array}\right|$ & 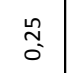 & $\left|\begin{array}{l}0 \\
0 \\
0 \\
0\end{array}\right|$ & $\left|\begin{array}{c}\hat{n} \\
0 \\
0\end{array}\right|$ & $\times$ & $\stackrel{\stackrel{n}{\sim}}{\sigma}$ & 目 & $\begin{array}{l}\stackrel{N}{N} \\
\text { O }\end{array}$ & $\left|\begin{array}{l}\stackrel{2}{2} \\
0\end{array}\right|$ & $\left|\begin{array}{c}\stackrel{\sim}{\approx} \\
0 \\
0\end{array}\right|$ & $\left|\begin{array}{c}\stackrel{N}{N} \\
0 \\
0\end{array}\right|$ & $\stackrel{\stackrel{n}{N}}{\sigma}$ & $\left|\begin{array}{l}8 \\
-i \\
-1\end{array}\right|$ & 啹 & $\left|\begin{array}{l}\hat{n} \\
\hat{0}\end{array}\right|$ & $\begin{array}{l}\text { ñ } \\
\text { o }\end{array}$ & $\left|\begin{array}{c}n \\
\hat{o}\end{array}\right|$ & $\left|\begin{array}{l}\stackrel{n}{2} \\
0\end{array}\right|$ & 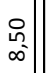 & $\left|\begin{array}{l}n \\
0 \\
0 \\
0 \\
0\end{array}\right|$ \\
\hline 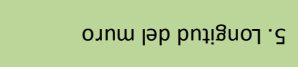 & $\begin{array}{l}n \\
\hat{\alpha} \\
0\end{array}$ & $\begin{array}{l}\frac{n}{a} \\
\sigma^{\prime}\end{array}$ & $\mid \begin{array}{l}0 \\
0 \\
0 \\
0\end{array}$ & $\times$ & $\begin{array}{l}n \\
\vdots \\
0\end{array}$ & $\stackrel{\stackrel{n}{\sim}}{\circ}$ & مُ & $\begin{array}{l}\text { 号 } \\
\text { o }\end{array}$ & $\left|\begin{array}{c}n \\
\sim \\
0\end{array}\right|$ & 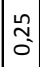 & $\left.\mid \begin{array}{c}n \\
\tilde{N} \\
0\end{array}\right]$ & $\stackrel{n}{\tilde{O}}$ & $\mid$ & : & $\mid \begin{array}{l}0 \\
0 \\
0 \\
0\end{array}$ & $\stackrel{\sim}{\sim}$ & $\stackrel{\sim}{\sim}$ & $\begin{array}{l}0 \\
0 \\
0 \\
0\end{array}$ & $\stackrel{n}{\sim}$ & $\left|\begin{array}{c}0 \\
0 \\
0 \\
0 \\
0\end{array}\right|$ \\
\hline 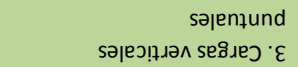 & $\left|\begin{array}{c|c}n \\
\hat{0} \\
0\end{array}\right|$ & 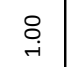 & $\times$ & $\mid \begin{array}{l}0 \\
0 \\
0 \\
0\end{array}$ & $\begin{array}{l}\text { 号 } \\
\text { on }\end{array}$ & $\stackrel{\stackrel{n}{\sim}}{0}$ & $\stackrel{\stackrel{n}{\sim}}{\circ}$ & 号 & $\left|\begin{array}{c}0 \\
0 \\
0 \\
0\end{array}\right|$ & $\left|\begin{array}{l}0 \\
0 \\
0 \\
0 \\
0\end{array}\right|$ & $\left|\begin{array}{c}0 \\
0 \\
0 \\
0\end{array}\right|$ & $\begin{array}{l}\text { in } \\
\text { in }\end{array}$ & $\mid$ & : & $\left|\begin{array}{l}0 \\
\text { hn } \\
0\end{array}\right|$ & $\stackrel{\sim}{\tilde{O}}$ & $\stackrel{\sim}{\sim}$ & 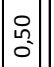 & $\underset{\infty}{\sim} \underset{\infty}{n}$ & $\begin{array}{l}1 \\
0 \\
0 \\
0\end{array}$ \\
\hline 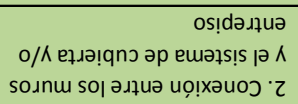 & {$\left[\begin{array}{c}n \\
\hat{0} \\
0\end{array}\right]$} & $x$ & $\left|\begin{array}{c}0 \\
\hdashline \\
-i\end{array}\right|$ & $\left|\begin{array}{l}\hat{n} \\
0 \\
0\end{array}\right|$ & $\begin{array}{l}\stackrel{n}{\sim} \\
0 \\
0\end{array}$ & $\stackrel{\stackrel{\sim}{N}}{0}$ & 雚 & 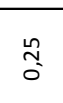 & $\left|\begin{array}{l}0 \\
0 \\
0 \\
0\end{array}\right|$ & $\left|\begin{array}{l}0 \\
0 \\
0 \\
0\end{array}\right|$ & 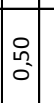 & 员 & {$\left[\begin{array}{c}\hat{n} \\
\hat{0}\end{array} \mid\right.$} & $\stackrel{\substack{n \\
0}}{0}$ & $\mid$ & $\begin{array}{l}\text { 目 } \\
\text { s. }\end{array}$ & $\begin{array}{l}\text { n̂. } \\
\text { co }\end{array}$ & $\left|\begin{array}{|l||}0 \\
0 \\
0 \\
0\end{array}\right|$ & $\begin{array}{l}\hat{n} \\
\infty \\
\infty\end{array}$ & $\left|\begin{array}{l}m \\
⿱ \\
0 \\
0 \\
0\end{array}\right|$ \\
\hline 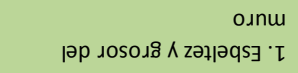 & $x$ & $\begin{array}{l}\stackrel{n}{2} \\
0 \\
0\end{array}$ & $\left|\begin{array}{l}n \\
\hat{0} \\
0\end{array}\right|$ & $\left|\begin{array}{l}n \\
\hat{\sigma} \\
0\end{array}\right|$ & $\begin{array}{l}n \\
\vdots \\
0\end{array}$ & 员 & مُ & 员 & 总 & 㞷 & 员 & $\stackrel{n}{\stackrel{n}{o}}$ & $\mid$ & $\begin{array}{l}\stackrel{n}{a} \\
0 \\
0\end{array}$ & $\left|\begin{array}{l}n \\
\hat{\sigma} \\
0\end{array}\right|$ & 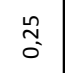 & 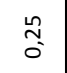 & 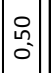 & $\begin{array}{l}0 \\
0 \\
0\end{array}$ & $\left|\begin{array}{l}n \\
0 \\
0 \\
0 \\
0\end{array}\right|$ \\
\hline$\frac{4}{\frac{4}{4}}$ & 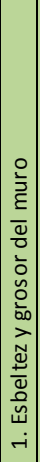 & 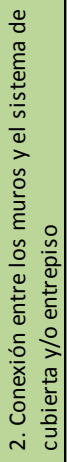 & 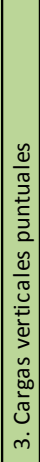 & 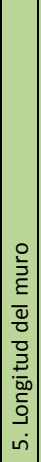 & 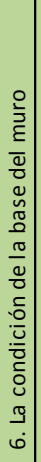 & 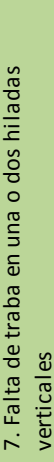 & 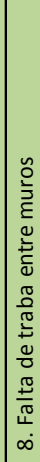 & 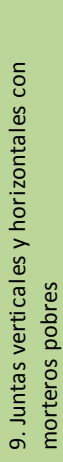 & 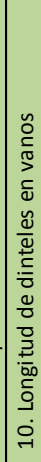 & 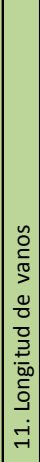 & 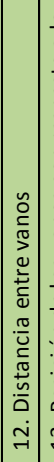 & 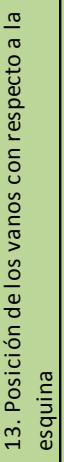 & 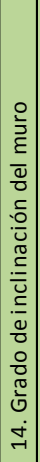 & 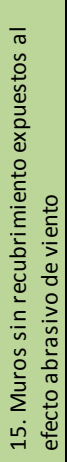 & 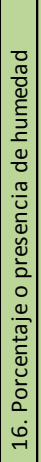 & 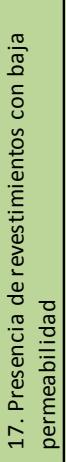 & 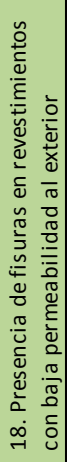 & 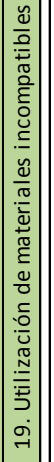 & 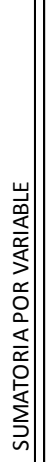 & 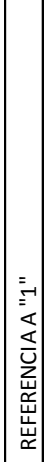 \\
\hline
\end{tabular}




\section{RESULTADOS Y DISCUSIONES}

\subsection{Evaluación del estado constructivo del muro de adobe}

El resultado determinado en la matriz de priorización representa la sumatoria para cada variable obteniendo un factor de incidencia o peso de esa variable en el conjunto de la matriz. Para normalizar los datos se relaciona la sumatoria de las variables a 1. En la Tabla 3, en la última fila de la tabla, se presentan los valores obtenidos.

En la matriz de priorización se consideran las 18 variables que representan los daños presentes en el muro de adobe; para completar la valoración o determinación integral del estado del muro analizado se incorpora la condición de si el muro es de carga y la magnitud, que para el presente caso se denomina Nivel de Gravedad. Para ello, y en base a los estudios de caso: Methodology for analysis, diagnosis and monitoring of damage in heritage architecture (earth and timber) in Cuenca, Ecuador. Case study Casa Peña in the Barranco of the city (Achig, 2010), así como a los resultados obtenidos en la aplicación del sistema de registro y diagnóstico de daños (SISREDA) desarrollado en el Proyecto vlirCPM (2007-2012), se determina que la incidencia en términos de afectación de estos factores pueden considerarse con el mismo peso que la consideración global de las diferentes variables que expresan los daños. Esto implica que el indicador integral que determina el estado del muro corresponde al $50 \%$, dado por las condiciones de daños; y, el otro $50 \%$ por los factores de condición si el muro es de carga y de la magnitud de los daños.

Al respecto, es importante mencionar que esta participación cualitativa es fruto del análisis de estudios de caso; y, por consiguiente corresponde a una hipótesis que debe someterse a estudios más específicos para determinar, de ser el caso, ajustes o complementaciones. De hecho, al momento, en el proyecto vlirCPM se está aplicando a diferentes estudios de caso la metodología de calificación expuesta en el presente artículo para contar con una base más amplia de sustento.

De acuerdo a lo comentado, se determinaron los siguientes valores para el Nivel de Gravedad (puntuación máxima $=50$ ): MURO CARGA (25): $\mathrm{NO}=0 ; \mathrm{SI}=25 ;$ MAGNITUD (25): $\mathrm{NO}=0$; $\mathrm{BAJA}=6,25 ;$ MEDIA $=12,5 ;$ ALTA $=18,75 ;$ MUY ALTA $=25$.

Los factores obtenidos en la matriz de priorización se utilizan para la determinación del indicador del Estado Constructivo del Muro de Adobe aplicando la siguiente relación:

$$
\mathbf{I E M A}=[(\mathrm{V} 1 * \mathrm{PP} 1)+(\mathrm{V} 2 * \mathrm{PP} 2)+(\mathrm{V} 3 * \mathrm{PP} 3)+\ldots \ldots . .(\mathrm{V} 18 * \mathrm{PP} 18)] / 2+\mathrm{GRV}
$$

IEMA: Indicador Integral del Estado constructivo de Muro de Adobe (en porcentaje).

V1 $\rightarrow$ V18: Valor cuantitativo (ver Tabla 2; valor entre 0 y 1).

PP1 $\rightarrow$ PP18: Factor de ponderación de cada variable (en porcentaje).

GRV: Nivel de Gravedad.

\subsection{Procedimiento para determinar el IEMA}

a) El equipo de trabajo, en base al análisis de campo, establece el valor de acuerdo a la escala de calificación (Tabla 2), de cada una de las variables elegidas para la valoración del muro de adobe. Esta fase es crítica, pues de ella depende la validez del análisis y la calificación; por ello, debe realizarse por evaluadores capacitados. En el caso de edificaciones o situaciones de mayor importancia es recomendable aplicar al menos dos análisis distintos realizados por igual número de evaluadores.

b) Una vez definidos los factores con sus respectivos grados, los evaluadores proceden a calificar cada una de las variables con el factor de ponderación.

c) Determinar el nivel o valor de Gravedad, para lo cual se establecerá si el muro de estudio es de carga o no, y la magnitud; es decir, la mayor o menor presencia de los daños identificados en términos de dimensiones o medidas; y

d) Establecer la calificación del muro de adobe, de acuerdo al IEMA: MUY ALTA > 75\%; ALTA de 50 a $75 \%$; MEDIA de 25 a $50 \%$; BAJA $<25 \%$; NO $=0$. 
El IEMA constituye una herramienta que permite establecer el nivel de afectación de un muro de adobe, lo cual sirve de base para la determinación de acciones ya sea de monitoreo, realización de estudios especializados o implementación de acciones emergentes. La calificación de un muro de adobe de acuerdo al IEMA se puede también traducir en los siguientes términos:

- MUY ALTA - Colapso: Cuando se ha superado el límite de estabilidad física del muro.

- ALTA - Crítico: El muro tiene un alto nivel de afectación, pero es susceptible de ser intervenido y recuperado.

- MEDIA - Estabilidad condicionada: El muro presenta daños, pero se mantiene la condición de estabilidad; por lo tanto se pueden realizar intervenciones para su recuperación.

- BAJA Y SIN AFECTACIÓN - Estable: Sin ninguna afectación.

\subsection{Determinación de alertas}

La determinación de alertas en un muro de adobe puede convertirse en una herramienta importante para el gestor, puesto que podría ser determinante para la toma de decisiones al intervenir en edificaciones patrimoniales. La presente investigación relaciona la determinación de alertas con el estado constructivo de un muro de adobe. En base a estas reflexiones, se determinó tres tipos de alertas:

- Nivel alto: el muro de adobe se encuentra en estado crítico y en el corto plazo podría colapsar y se requiere intervenir de forma inmediata.

- Nivel medio: el muro se encuentra en estado estable pero condicionado a la evolución de los daños presentes. El daño se podría estabilizar y se requiere monitorear con una frecuencia semestral.

- Nivel bajo: el muro se encuentra completamente estable y no necesita una intervención.

\subsection{Determinación de acciones a seguir en muros de adobe}

Una vez determinadas las alertas es importante tomar decisiones sobre las acciones a seguir.

- INTERVENCIÓN. Una vez que se ha detectado una alerta alta se deben controlar los factores que hacen que los daños evolucionen de una manera rápida, afectando a la estabilidad y pueden llevar a la estructura al colapso. En este caso se deben hacer obras emergentes. Por ejemplo, si un factor constituye la falta de conexión del muro con la cubierta, resulta necesario colocar una viga collar para evitar la presencia de cargas puntuales. Si se tiene humedad en un muro hay que controlarla o colocar una cubierta provisional de ser el caso. El asunto es estabilizar el muro y detener el deterioro.

- MONITOREO. Cuando el muro de adobe no evidencia ningún estado o situación de gravedad o está en un estado medianamente estable, entonces debe ser monitoreado a corto, mediano o largo plazo dependiendo de la gravedad del daño. El tema de monitoreo es fundamental para la gestión del patrimonio y debería ser estudiado a profundidad en estudios especializados.

La metodología planteada permite determinar el estado constructivo de un muro de adobe, así como las acciones a seguir dependiendo de su calificación. Sin embargo, se deberían desarrollar en futuras investigaciones, otros modelos complementarios para los diferentes elementos constitutivos, sobre todo, referentes a la estructura de un bien patrimonial, cuya integración determinará el estado constructivo de toda la edificación. La presente investigación, solamente constituye una parte de todo este proceso. Por motivos prácticos de aplicación del modelo en las edificaciones patrimoniales del Centro Histórico de Cuenca, se podría priorizar su aplicación en muros de adobe de gran importancia por su magnitud, proporciones, relevancia estructural o en aquellos muros que puedan influir en el comportamiento de ambientes aledaños o en toda la edificación. También se podría aplicar el modelo dando prioridad a muros de adobe de valor excepcional, como en el caso de muros que contienen pintura mural. 


\section{CONCLUSIONES}

La investigación propone el diseño de un registro de daños para determinar el estado constructivo de muros de adobe, lo cual resulta esencial para la conservación del patrimonio edificado, en particular de la ciudad de Cuenca. Se enfoca el tema de daños con una visión preventiva. Si se conocen los factores relacionados con un daño, así como su posible evolución, se podrá intervenir antes de que llegue a un punto crítico o de colapso; así también, el sistema propuesto establece condiciones para implementar procesos de monitoreo y control. La presente investigación constituye una herramienta que apoya a la conservación y gestión del patrimonio, ayudando al gestor en la toma oportuna de decisiones. El sistema alerta cuando se debe actuar, especialmente en condiciones críticas.

El diseño del sistema de análisis de muros de adobe tiene como propósito fundamental determinar el estado constructivo integral de este elemento; para ello, se parte de la determinación de los principales daños, en términos de la frecuencia que se encuentran en los muros, así como su incidencia en la estabilidad. Debido a que en un muro pueden presentarse de manera concurrente un conjunto de situaciones que lo afectan, el sistema planteado emplea el análisis multivariado; así, el indicador integral del estado (IEMA) se obtiene mediante una valoración ponderada en la que intervienen las diferentes afectaciones así como el nivel de gravedad, el mismo que está en función de la magnitud de los daños y si el muro de adobe es de carga o no. El análisis de un muro específico da como resultado la calificación en una escala de 5 estados de afección: Muy alta, Alta, Media, Baja y Sin afección; estos valores a su vez dan lugar a recomendaciones de actuación, de tal manera que se implementen acciones para la conservación del elemento constructivo y por ende de la edificación patrimonial.

La obtención de resultados apropiados en la calificación del estado constructivo de muros de adobe depende en gran medida de la calidad de la obtención de los datos, por lo que es importante que se cuente con personal capacitado para el efecto. De igual manera, teniendo en cuenta que el sistema tiene el propósito de generar alertas tempranas, se debe implementar un apropiado proceso de monitoreo y con ello contribuir a mitigar o impedir que se produzcan acontecimientos que afecten la conservación del bien patrimonial. La práctica del monitoreo es muy restringida o inexistente en nuestro medio; por esta razón las edificaciones, incluso las más importantes, presentan afecciones que requieren de acciones importantes para su remediación; consecuentemente, los proyectos deben incluir recursos destinados a la implementación de un proceso de vigilancia y reporte para un adecuado y pertinente seguimiento y control. En este sentido, el modelo planteado puede constituirse en una herramienta que facilite, sistematice y ayude a determinar en diferentes momentos el estado y la evolución de la condición de los muros; por consiguiente, también de la edificación.

\section{AGRADECIMIENTOS}

Nuestro agradecimiento al Dr. Jan Feyen por su apoyo invalorable en la conclusión de este artículo. Agradecemos a los miembros del proyecto vlirCPM de la Facultad de Arquitectura y Urbanismo de la Universidad de Cuenca, por facilitar información para la realización del presente trabajo, en especial a su director, el arquitecto Fausto Cardoso y a la arquitecta Verónica Heras por nutrir el artículo con sus valiosos comentarios. Agradecemos al sociólogo Lucas Achig por su aporte en la redacción y corrección de estilo. Un reconocimiento a la memoria del arquitecto Marcelo Zúñiga, coautor de este artículo, para quien mi más sincero agradecimiento por su invalorable aporte a la investigación.

\section{BIBLIOGRAFÍA}

Achig, M.C., 2010. Methodology for analysis, diagnosis and monitoring of damage in heritage architecture (earth and timber) in Cuenca, Ecuador. Case Study: Casa Peña in theBarranco of the city. Tesis de Maestría en Conservación de Monumentos y Sitios, Universidad Católica de Lovaina - Universidad de Cuenca, Leuven, Bélgica, 11-12, 180-249. 
Achig, M.C., M.C. Paredes, 2001. Arqueología del color: Historia, mundo y significación. Estudio y propuesta para el Centro Histórico de Cuenca. Tesis previa a la obtención del título de arquitecto, Universidad de Cuenca, Facultad de Arquitectura y Urbanismo, Cuenca, Ecuador, 13-33.

Blondet, M., G.V. Garcia M., S. Brzev, A. Rubiños, 2003. Construcciones de adobe resistentes a los terremotos. Publicado como una contribución a la Enciclopedia Mundial de Vivienda del EERI/IAEE, 13 pp. Descargado de www.world-housing.net/wp-content/uploads/ 2011/06/Adobe_Tutorial_Spanish_Blondet.pdf en agosto del 2013.

Brown, P.W., J. R. Clifton, 1978. Adobe. 1: The properties of adobe. Stud. Conserv., 23(4), 139-146.

Campos, F., 2009. Análisis de bloques de adobe. Municipalidad de Cuenca, Laboratorio de Suelos, Cuenca, Ecuador. Comunicación personal.

Carreño, M.L., 2006. Técnicas innovadoras para la evaluación del riesgo sísmico y su gestión en centros urbanos: Acciones ex ante y ex post. Tesis doctoral de la Universidad Politécnica de Cataluña, Escuela Técnica Superior de Ingenieros de Caminos, Canales y Puertos, Barcelona, España, 158-176.

De Jongh, S., M. Van Wijnendaele, 2009. Earthquakes and other damaging mechanisms to earth structures, Cuenca, Ecuador. Tesis para obtener el título de ingenieros, Universidad Católica de Lovaina - Universidad de Cuenca, Cuenca, Ecuador, 1-26, 44-45.

De Jongh, S., M. Van Wijnendaele, F. Cardoso, K. Van Balen, 2009. Damage atlas of historical Cuenca, Ecuador. Damage patterns found in (mainly earthen) building materials. VLIR-IUOS, Universidad Católica de Lovaina - Universidad de Cuenca, Cuenca, Ecuador, 75 pp. Descargado de http://www.slideshare.net/mvanwijnendaele/damage-atlas-historical-cuenca en julio del 2013.

Espinoza, P., I. Calle, 2002. La cité cuencana - El afrancesamiento de Cuenca en la época republicana (1860-1940). Facultad de Arquitectura de la Universidad de Cuenca, Cuenca, Ecuador, 29-33.

Fratini, F., E. Pecchioni, L. Rovero, U. Tonietti, 2011. The earth in the architecture of the historical centre of LameziaTerme (Italy): Characterization for restoration. Appl. Clay Sci., 53, 519-516.

García, B., 2000. Región y lugar en la arquitectura latinoamericana contemporánea. Centro Editorial Javeriano (CEJA), Bogotá, Colombia, 57-58.

Jaramillo, D., 2004. Del plano del damero a la ciudad del migrante. Tomado del libro: Cuenca - Santa Ana de las Aguas. Ediciones Libri Mundi, Quito, Ecuador, 94 pp.

Jiménez, M.C., I. Cañas, 2007. The selection of soils for unstabilised earth building: A normative review. Construct. Build. Mater., 21, 237-251.

I. Municipalidad de Cuenca, 2010. Actualización del inventario de las edificaciones patrimoniales del Centro Histórico de Cuenca. Base de datos del inventario a nivel de registro en formato digital. Municipalidad de Cuenca, Cuenca, Ecuador.

Houben, H., H. Guillaud, 1989. Earth construction: A comprehensive guide. CRATerre-EAG, Intermediate Technology Publications, Marseille, Francia, 146 pp.

Leroy Tolles, E., E.E. Kimbro, W.S. Ginell, 2002. Guías de planeamiento e ingeniería para la estabilización sismorresistente de estructuras históricas de adobe. The Getty Conservation Institution, Los Ángeles, USA, 160 pp. Descargado de http://www.getty.edu/conservation/ publications_resources/pdf_publications/pdf/seismic_retrofitting_esp.pdf en julio del 2013.

Malczewski, J., 1999. Gis and multicriteria decision analysis. John Wiley \& Sons Inc., 393 pp.

Minke, G., 2001. Manual de construcción en tierra: La tierra como material de construcción y su aplicación en la arquitectura tradicional. Editorial Nordan-Comunidad, Uruguay, 222 pp.

Nara Document on Authenticity, 1994, UNESCO - ICCROM-ICOMOS, Japan. Descargado de http://whc.unesco.org/archive/nara94.htm en julio del 2013.

Pacheco-Torgal, F., S. Jalali, 2011. Earth construction: Lessons from the past for future eco-efficient construction. Construct. Build. Mater., 29, 512-519.

Paolini, A., A. Vafadari, G. Cesaro, M. Santana Quintero, K. Van Balen, O. Vileikis, L. Fakhoury, 2012. Risk management at heritage sites. A case study of the Petra world heritage site. UNESCO Katholieke Universiteit Leuven, Ammán, Jordania, 172 pp. 
Piedra, D., 2008. Patologías y sus causas en muros en tierra cruda y cocida en las edificaciones patrimoniales. Tesis de Maestría en Conservación de Monumentos y Sitios, Facultad de Arquitectura y Urbanismo, Universidad de Cuenca, Cuenca, Ecuador, 81 pp.

Proyecto: vlirCPM World Heritage City Preservation Management, 2009. El registro de daños de la edificación patrimonial en la ciudad de Cuenca. Documento no publicado. Facultad de Arquitectura y Urbanismo, Universidad Católica de Lovaina - Universidad de Cuenca, Cuenca, Ecuador, $18 \mathrm{pp}$.

Proyecto vlirCPM World Heritage City Preservation Management, 2010. Atlas de daños. Edificaciones Patrimoniales de Cuenca. Facultad de Arquitectura y Urbanismo, Universidad Católica de Lovaina - Universidad de Cuenca, Cuenca, Ecuador, 12 pp \& anexos.

Proyecto vlirCPM World Heritage City Preservation Management, 2007-2012. SISREDA - Sistema de registro y diagnóstico de daños aplicado en los proyectos de intervención en los edificios: Antiguo seminario San Luis, Escuela Central La Inmaculada, Casa del Buen Pastor. Facultad de Arquitectura y Urbanismo, Universidad Católica de Lovaina - Universidad de Cuenca, Cuenca, Ecuador, $28 \mathrm{pp}$.

Rivera, N., M. Rivera, 2001. Hermano Juan Bautista Stiehle Redentorista: Testimonio. Editorial Amazonas S.A., Cuenca, Ecuador, 58 pp.

Rotondaro, R., 2011. Adobe. En: Neves, C., O. Borges Faria (Eds.), Técnicas en construcción con tierra. Red Iberoamericana PROTERRA, Bauru, Brasil, 16-25. Descargado de http://issuu.com/ julianadan/docs/construccioncontierra en julio del 2013.

Van Balen, K, 2007. Damage and monitoring. Presentación para el proyecto vlirCPM: World Heritage City Preservation Management. Facultad de Arquitectura y Urbanismo, Universidad Católica de Lovaina - Universidad de Cuenca, Cuenca, Ecuador, Diapositiva 2. 